\title{
Emergency Abdominal Surgery Outcomes of Critically Ill Patients on Extracorporeal Membrane Oxygenation: A Case-Matched Study with a Propensity Score Analysis
}

\author{
Anna Taieb $^{1} \cdot$ Florence Jeune $^{2} \cdot$ Said Lebbah $^{3} \cdot$ Matthieu Schmidt $^{4,5} \cdot$ Romain Deransy $^{6}$ • \\ Jean-Christophe Vaillant ${ }^{2}$ - Charles-Edouard Luyt ${ }^{4,5}$ - Christophe Trésallet ${ }^{1,7}$. \\ Alain Combes ${ }^{4,5} \cdot$ Nicolas Bréchot ${ }^{4,8}$
}

Published online: 4 February 2019

(C) Société Internationale de Chirurgie 2019

\begin{abstract}
Background Extracorporeal membrane oxygenation (ECMO) induces coagulation disorders increasing the risk of bleeding during invasive procedures. We aimed to describe the outcomes of critically ill ECMO patients undergoing emergency abdominal surgery compared to non-ECMO patients.

Study design This is a retrospective case-matched single-center study with propensity score analysis in a tertiary ICU. All patients who underwent abdominal surgery were included.

Results From 2006 to 2014, 77 patients admitted in our ICU underwent emergency abdominal surgery, 35 were on ECMO. Surgery indications were comparable for ECMO and non-ECMO patients: mostly intestinal ischemia (42\%) and cholecystectomy $(25 \%)$. Postoperative bleeding was significantly more frequent in ECMO group versus nonECMO: $77 \%$ versus $40 \%$ transfused, with medians of $13(6-22)$ versus $3(0-5)$ packed red blood cell; 9 (3-17) versus $0(0-4)$ fresh frozen plasma and $12(3-22)$ versus $0(0-8)$ platelet units $(p<0.001$ for all items). Reintervention for hemorrhage was required in $20 \%$ versus $2 \%$, respectively, $p=0.02$. At multivariable analysis, ECMO was strongly associated with bleeding (OR, 5.6 [95\% CI, 2.0-15.4]; $p=0.001)$. ICU mortality was higher for ECMO-treated patients $(69 \%$ vs. $33 \%$; $p=0.003)$, but perioperative mortality remained comparable between groups $(11 \%$ vs. $12 \%$, NS). Propensity score-matched analysis confirmed more frequent and severe bleeding in ECMO patients.

Conclusions Abdominal surgery procedures on ECMO-treated patients are associated with a higher risk of hemorrhage compared to non-ECMO ICU patients. Further studies are needed to optimize ECMO patient management during such interventions.
\end{abstract}

Electronic supplementary material The online version of this article (https://doi.org/10.1007/s00268-019-04930-2) contains supplementary material, which is available to authorized users.

Christophe Trésallet

christophe.tresallet@aphp.fr

1 Department of Digestive and Endocrine Surgery, Hôpital Pitié-Salpêtrière, Assistance Publique-Hôpitaux de Paris, Sorbonne University (Paris 6), Paris, France

2 Department of Digestive and Hepato-Pancreato-Biliary Surgery, Hôpital Pitié-Salpêtrière, Assistance PubliqueHôpitaux de Paris, Sorbonne University (Paris 6), Paris, France
3 Clinical Research Department, Hôpital Pitié-Salpêtrière, Assistance Publique-Hôpitaux de Paris, Sorbonne University (Paris 6), Paris, France

4 Medical-Surgical ICU, Hôpital Pitié-Salpêtrière, Assistance Publique-Hôpitaux de Paris, Sorbonne University (Paris 6), Paris, France

5 INSERM, UMRS_1166-iCAN, Institute of Cardiometabolism and Nutrition, Paris, France 


$\begin{array}{lll}\text { Abbreviations } & \\ \text { ARDS } & \text { Acute respiratory distress syndrome } \\ \text { ECMO } & \text { Extracorporeal membrane oxygenation } \\ \text { PRBCs } & \text { Packed red blood cell units } & \\ \text { SAPS } & \text { Simplified Acute Physiology Score } \\ \text { SOFA } & \begin{array}{l}\text { Sepsis-related organ failure assessment } \\ \text { VA-ECMO }\end{array} & \begin{array}{l}\text { Venoarterial-extracorporeal } \\ \text { oxygenation }\end{array} \\ \text { VV-ECMO } & \begin{array}{l}\text { Venovenous-extracorporeal } \\ \text { oxygenation }\end{array} & \text { membrane } \\ & & \end{array}$

\section{Introduction}

Extracorporeal membrane oxygenation (ECMO) is increasingly used as rescue therapy during major cardiac failure or acute respiratory distress syndrome (ARDS) [1-3], but remains associated with major complications. ECMO-treated patients are at high risk of hemorrhage during invasive procedures, due to major coagulation disorders induced by the patient's critical state and the device itself [4-6]. In this context, emergency surgery, when needed, is perceived as extremely detrimental. The outcomes of ECMO-assisted patients undergoing noncardiac surgery have only been poorly described to date. More specifically, no study focused on abdominal surgery in this setting. This may be of particular interest in order to improve perioperative management of these patients.

The aim of the present case-matched study, using a propensity score analysis, was to assess the outcomes of patients on ECMO who required emergency abdominal surgery and to compare the postoperative outcomes with those of non-ECMO patients matched for initial severity.

\section{Methods}

\section{Patients and data collection}

All our intensive care unit (ICU) patients who underwent emergency abdominal surgery between July 2006 and

6 Department of Anesthesiology and Critical Care, Hôpital Pitié-Salpêtrière, Assistance Publique-Hôpitaux de Paris, Sorbonne University (Paris 6), Paris, France

7 INSERM-CNRS U 678- laboratoire d'imagerie Biomédicale (LIB), Departement of General, Visceral and Endocrine Surgery, Groupe Hospitalier Pitié-Salpêtrière, Sorbonne University (Paris 6), 47/83, Boulevard de l'Hôpital, 75651 Paris Cedex 13, France

8 INSERM U1050, Centre Interdisciplinaire de Recherche en Biologie, College de France, 11, Place Marcelin-Berthelot, Paris, France
August 2014 were eligible for the study. Patients were screened from the operating room registry of the surgery department. Data were extracted from the computerized ICU charts. In accordance with our hospital's Institutional Review Board (Committee for the Protection of Human Subjects) ethical standards, informed consent for demographic, physiological and hospital-outcome data analyses was not required because this observational study did not modify existing diagnostic or therapeutic strategies.

\section{ECMO implantation and management}

All patients underwent Doppler echocardiography to evaluate hemodynamic status before ECMO implantation. Venoarterial-ECMO (VA-ECMO) was indicated for refractory cardiogenic shock, defined as evidence of tissue hypoxia (e.g., extensive skin mottling or elevated blood lactate) associated with low cardiac index $(<2.2 \mathrm{~L} / \mathrm{min} /$ $\mathrm{m}^{2}$ ), despite infusion of high catecholamine dose and adequate fluid loading [7]. Venovenous-ECMO (VVECMO) was indicated for ARDS refractory to conventional treatment [3].

Management of ECMO-implanted patients in our ICU was described previously $[8,9]$. All VA-ECMO devices were surgically inserted with femoral-femoral $23 \mathrm{~F}$ to $29 \mathrm{~F}-$ $15 \mathrm{~F}$ to $18 \mathrm{~F}$ cannulation. An additional $7 \mathrm{~F}$ catheter was systematically inserted into the femoral artery to prevent leg ischemia. Pump speed was adjusted to obtain blood flow of $3.5-4.5 \mathrm{~L} / \mathrm{min}$. Patients were assessed daily for possible ECMO weaning using clinical and echocardiographic criteria, as previously described [10]. VV-ECMO was inserted percutaneously with femoral-jugular $25 \mathrm{~F}$ to $29 \mathrm{~F}-18 \mathrm{~F}$ to $21 \mathrm{~F}$ cannulation. Weaning was achieved as previously described [9]. Experienced perfusionists checked the circuit daily.

A 5000-IU heparin bolus was injected at ECMO initiation, and intravenous (IV) unfractionated heparin was given to maintain the activated partial thromboplastin time (aPTT) ratio at 1.5-2 times normal. The heparin dose was adapted at least once daily. Heparin was stopped when bleeding occurred or the platelet count decreased significantly $(<50 \mathrm{G} / \mathrm{L})$ [11]. The membrane oxygenator was changed prophylactically when the platelet count decreased significantly $(<50 \mathrm{G} / \mathrm{L})$, blood oxygenation declined sharply or significant hemolysis appeared [12].

\section{Abdominal surgery}

All procedures were performed by trained abdominal surgeons. Cholecystectomy used retrograde or anterograde (when pediculitis was too severe) techniques.

For small bowel or colon ischemia, the length of intestinal resection depended on the extension of ischemic 
lesions. The mesentery or mesocolon was ligated with a thermo-fusion instrument (Ligasure ${ }^{\circledR}$, Covidien, Minneapolis, Minnesota, USA), and intestinal stoma was created with $3.0 \mathrm{Vicryl}^{\circledR}$. Bowel anastomoses when performed used a 65 or 80 GIA $^{\circledR}$ stapler (AutoSuture Company, United States Surgical Corporation, Norwalk, CT).

\section{Outcome definitions}

The following definitions were applied:

Preoperative period: the $24 \mathrm{~h}$ preceding surgery; immediate postoperative period: the 24-h postsurgery; and late postoperative period: the time between the $24-\mathrm{h}$ postsurgery and ICU discharge or death; perioperative bleeding: the need of transfusion of at least three units of packed red blood cell units (PRBCs) per procedure and within the 72-h postsurgery.

\section{Statistical analyses}

Continuous variables, expressed as medians (first-third interquartile range $[\mathrm{IQR}]$ ), were compared with MannWhitney $U$ tests. Categorical variables, expressed as $n(\%)$, were compared with Fisher's exact test. For multivariable analyses of factors associated with perioperative bleeding, all those associated with the dependent variable in univariable analyses with $p<0.2$ were included using a backward stepwise logistic-regression model. For practical purposes, continuous variables were transformed into categorical variables, with the median as the threshold (except for the sepsis-related organ failure assessment (SOFA) score which was trichotomized). All potential explanatory variables included in the multivariable analyses were subjected to a correlation matrix for analysis of collinearity, and variables associated with one another were not included in the model. Thereafter, multiple backward stepwise logistic-regression analyses eliminated variables with an exit threshold set at $p>0.10$.

For sensitivity analyses, baseline characteristics of ECMO and non-ECMO patients were matched by their propensity scores to receive ECMO [13]. Baseline covariates (age, sex, body mass index, sepsis, underlying diagnosis, Simplified Acute Physiology Score (SAPS) II and SOFA score) were used to build an initial logistic-regression model predicting ECMO implantation. After calculating each patient's propensity score to be ECMOimplanted, ECMO recipients were matched (1:1) to nonECMO ICU patients for the analysis, without replacement, based on the propensity score log odds ("logit"). Using the estimated logits, a randomly selected ECMO patient was matched to the non-ECMO patient with the closest estimated logit value with a maximum $20 \%$ difference between the two logits. Investigators were blinded to other patient data during the matching procedure.

Analyses were computed with SPSS v20 statistics package (IBM Corporation, Armonk, NY), Prism 4.0c software (GraphPad Software, La Jolla, CA), SAS v9.3 (SAS Institute Inc, Cary, NC) and R software (R foundation for statistical computing, Vienna, Austria), with $p<0.05$ defining significance.

\section{Results}

\section{Study population}

Among 1166 ECMO-implanted patients during the study period, $35(3 \%)$ required abdominal surgery (Fig. 1). Indications for ECMO in this population are reported in Table 1. The two main indications for surgery were digestive ischemia and acute cholecystitis in 15 and eight patients, respectively. The other indications are reported in Supplementary Table 1. Patients' demographic characteristics are given in Table 1. The cohort consisted predominantly of VA-ECMO patients implanted for cardiogenic shock, who required secondary surgery for abdominal complications. Only $20 \%$ were admitted primarily for gastrointestinal disease. Patients were young and very severely ill, as reflected by the median SOFA score of 16 and the median SAPS II of 71 at admission. Forty-two nonECMO patients underwent abdominal surgery during the study period. They had the same underlying diagnoses and indications for surgery profiles as the ECMO-treated patients, but were older and slightly less severely ill.

\section{Perioperative management}

Patients' preoperative status is described in Table 2. Both groups had comparable hemodynamic impairments. Notably, the ECMO group had thrombocytopenia and lower fibrinogenemia than non-ECMO patients. Surgical characteristics are reported in Table 2 . Surgery was predominantly cholecystectomy, digestive resection and stoma creation via laparotomy. A significantly higher percentage of ECMO patients $(66 \%)$ experienced bleeding during surgery requiring transfusion than non-ECMO patients (48\%). Perioperative bleeding (defined as transfusion of $>3$ PRBCs during the first 72-h postsurgery) was significantly more frequent for ECMO-treated patients $(74 \%$ vs $33 \%, p<0.001)$.

\section{Outcomes}

ECMO patients' postoperative periods were marked by significantly more bleeding than non-ECMO patients 


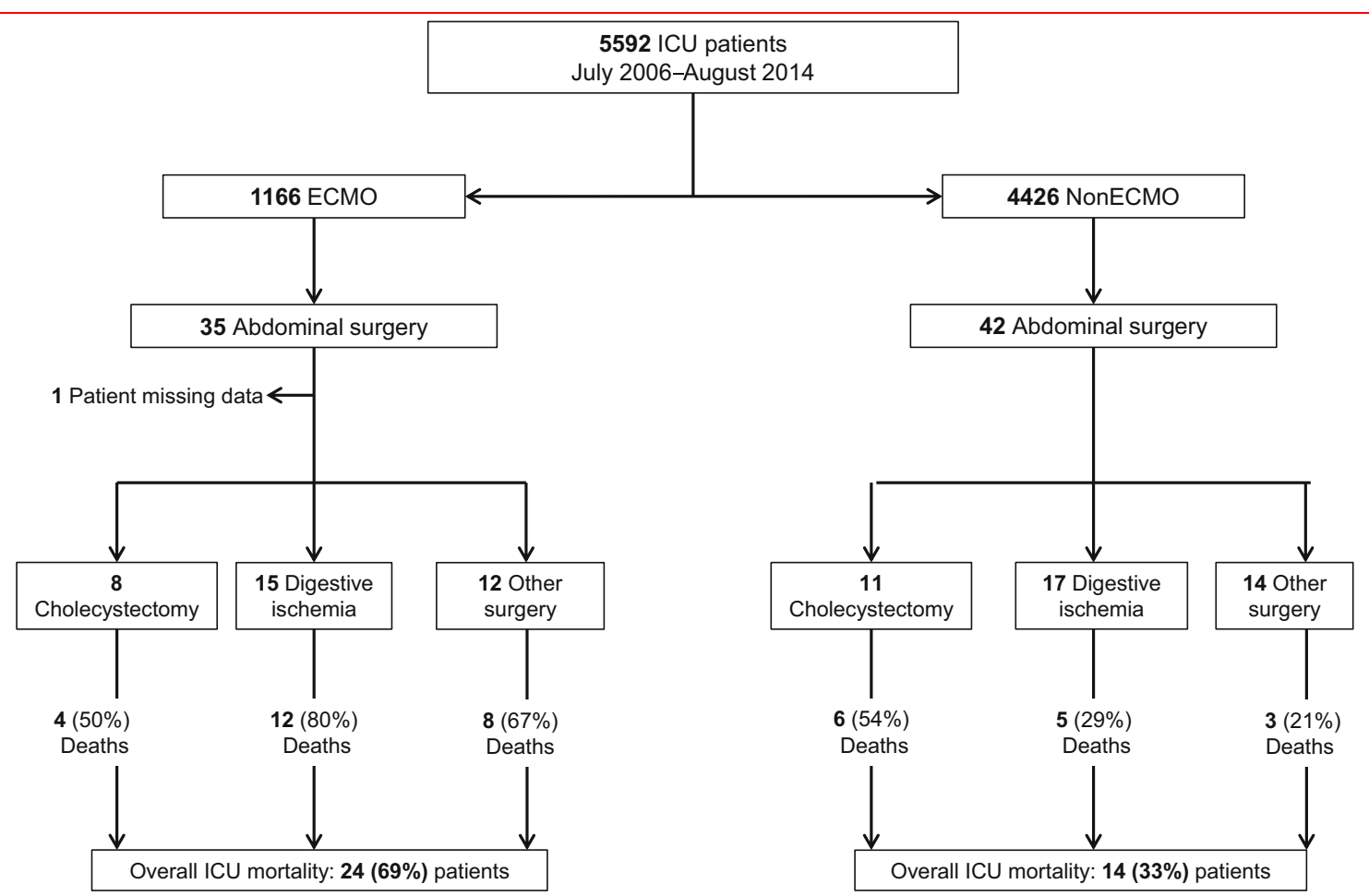

Fig. 1 Study flowchart. ICU intensive care unit, ECMO extracorporeal membrane oxygenation

(Table 3), with $77 \%$ versus $40 \%$ receiving PRBC, fresh frozen plasma or platelet units ( $p<0.001$ for all items). Reintervention for hemorrhage was significantly more frequent in the ECMO group (20\%) than in the non-ECMO group $(2 \% ; p=0.02)$. Importantly, bleeding complication rates were comparable for VA-ECMO and VV-ECMOtreated patients (Supplementary Table 2).

Perioperative mortality ( $<72$-h postsurgery) was comparable between groups (Table 3): four patient in ECMOtreated group (three died of refractory multiorgan failure after cardiogenic shock with digestive ischemia and one of hemorrhagic shock following abdominal necrosectomy for acute pancreatitis) and three in the non-ECMO group (all with episodes of refractory multiorgan failure following cardiogenic shock with digestive ischemia and two with secondary ventilator-associated pneumonia). Five (14\%) ECMO group patients' deaths were directly triggered by abdominal surgery complications (one with postoperative peritonitis, and four with hemorrhagic shock following bleeding at the surgical site) versus one (2\%) non-ECMO patient (postoperative peritonitis) $(p=0.08)$. Finally, 24 (69\%) ECMO-treated patients died in-ICU versus 14 (33\%) non-ECMO patients $(p=0.003)$. ECMO group deaths were distributed as follows: ten of refractory multiorgan failure, five of hemorrhagic shock, four of ventilator-associated pneumonia, three without myocardial recovery and limitation of active therapeutics, one without pulmonary recovery and one of peritonitis. In the non-ECMO group, refractory multiorgan failure accounted for seven inICU deaths, ventilator-associated pneumonia for two, the absence of neurological recovery for two, peritonitis for one and hemorrhagic shock for two. ICU mortality reached $80 \%$ for ECMO patients treated surgically for digestive ischemia and $50 \%$ of patients undergoing cholecystectomy.

\section{Factors associated with postoperative bleeding}

At univariable analysis, age, body mass index, SOFA score and ECMO implantation were associated with perioperative bleeding, whereas laparotomy was associated with a protective effect (Supplementary Table 3). At multivariable analysis ECMO was independently associated with a 5.6-fold increased risk of bleeding, while preoperative SAPS II $>70$ increased it by 2.9 (Fig. 2).

For sensitivity analyses, 21 ECMO patients could be matched to 21 non-ECMO patients based on their propensity scores to be ECMO-implanted. Characteristics of matched and unmatched patients are given in Supplementary Table 4 . Propensity matching yielded two groups of highly comparable patients in terms of underlying diagnosis, demographic characteristics, type of abdominal procedure and preoperative disease severity (Tables 1,2 ). A markedly higher rate of perioperative bleeding was confirmed for ECMO patients compared to matched nonECMO controls and the notably higher amounts of blood products received by the former. 
Table 1 Patient characteristic at ICU admission

\begin{tabular}{|c|c|c|c|c|c|c|}
\hline \multirow[t]{2}{*}{ Characteristic } & \multicolumn{2}{|c|}{ Unmatched patients $(n=77)$} & \multirow[t]{2}{*}{$p$} & \multicolumn{2}{|c|}{ Propensity-matched patients $(n=42)$} & \multirow[t]{2}{*}{$p$} \\
\hline & $\operatorname{ECMO}(n=35)$ & non-ECMO $(n=42)$ & & $\operatorname{ECMO}(n=21)$ & non-ECMO $(n=21)$ & \\
\hline \multicolumn{7}{|l|}{ Demographic } \\
\hline Year & $\begin{array}{l}2012 \\
(2011-2013)\end{array}$ & $\begin{array}{l}2010 \\
(2008-2012)\end{array}$ & 0.009 & $\begin{array}{l}2012 \\
(2010-2012)\end{array}$ & $\begin{array}{l}2010 \\
(2009-2012)\end{array}$ & 0.14 \\
\hline Age (years) & $59(43-64)$ & $64(54-74)$ & 0.02 & $61(51-64)$ & $55(51-63)$ & 0.6 \\
\hline Males & $27(77)$ & $28(67)$ & 0.45 & $14(67)$ & $14(67)$ & 1.0 \\
\hline Body mass index $\left(\mathrm{kg} / \mathrm{m}^{2}\right)$ & $27(24-32)$ & $27(22-29)$ & 0.28 & $26(23-31)$ & $26(23-30)$ & 0.8 \\
\hline Charlson score & $2(1-3)$ & $3(2-4)$ & 0.09 & $2(1-3)$ & $2(1-4)$ & 0.5 \\
\hline McCabe and Jackson score & $2(1-3)$ & $1.5(1-2)$ & 0.76 & $2(1-3)$ & $1(1-2)$ & 0.6 \\
\hline Immunocompromised & $6(17)$ & $15(36)$ & 0.08 & $5(24)$ & $6(29)$ & 1.0 \\
\hline \multicolumn{7}{|l|}{ Reason for ICU admission } \\
\hline Postoperative & $19(54)$ & $19(45)$ & 0.50 & $11(52)$ & $11(52)$ & 1.0 \\
\hline Sepsis & $13(37)$ & $20(48)$ & 0.82 & $9(43)$ & $8(38)$ & 1.0 \\
\hline \multicolumn{7}{|l|}{ Diagnosis $^{\mathrm{a}}$} \\
\hline Cardiovascular & $18(51)$ & $15(36)$ & 0.18 & $11(52)$ & $12(57)$ & 1.0 \\
\hline Gastrointestinal & $7(20)$ & $9(21)$ & 1.00 & $5(24)$ & $3(14)$ & 0.7 \\
\hline Metabolic & 0 & $1(2)$ & 1.00 & 0 & $1(5)$ & 1.0 \\
\hline Neurologic & 0 & $3(7)$ & 0.25 & 0 & $2(10)$ & 0.5 \\
\hline Renal or genitourinary & $1(3)$ & $6(14)$ & 0.12 & 0 & $1(5)$ & 1.0 \\
\hline Respiratory & $11(31)$ & $12(29)$ & 0.81 & $5(24)$ & $5(24)$ & 1.0 \\
\hline SOFA score & $16(12-17)$ & $11(7-14)$ & $<0.001$ & $15(10-17)$ & $14(10-15)$ & 0.4 \\
\hline SAPS II & $71(60-83)$ & $68(54-86)$ & 0.76 & $66(58-82)$ & $72(56-88)$ & 0.7 \\
\hline \multicolumn{7}{|l|}{ ECMO indication $^{\mathrm{a}}$} \\
\hline Cardiogenic shock & $22(63)$ & - & - & $16(76)$ & - & - \\
\hline Cardiomyopathy & $8(23)$ & - & - & $6(29)$ & - & - \\
\hline Septic shock & $5(14)$ & - & - & $5(24)$ & - & - \\
\hline Myocardial infarction & $4(11)$ & - & - & $2(10)$ & - & - \\
\hline Cardiac arrest & $3(9)$ & - & - & $2(10)$ & - & - \\
\hline Other & $2(6)$ & - & - & $1(5)$ & - & - \\
\hline ARDS & $13(37)$ & - & - & $5(24)$ & - & - \\
\hline \multicolumn{7}{|l|}{ ECMO hook-up } \\
\hline Venoarterial & $22(63)$ & - & - & $16(76)$ & - & - \\
\hline Venovenous & $13(37)$ & - & - & $5(24)$ & - & - \\
\hline
\end{tabular}

Results are expressed as number (\%) or median (first-third IQR)

SAPS Simplified Acute Physiology Score, SOFA sepsis-related organ failure assessment, ARDS acute respiratory distress syndrome

Significant differences are in bold type

${ }^{\mathrm{a}}$ Several may be involved

-Not applicable

\section{Discussion}

In this study, we assessed the outcomes of patients on ECMO who required emergency abdominal surgery. We found that, although such invasive procedures were feasible, they were associated with a significantly increased risk of bleeding compared to non-ECMO ICU patients.
To date, outcomes of abdominal surgery under ECMO have not been reported. Up to $30 \%$ of ECMO-treated patients experience bleeding complications, questioning the feasibility of these invasive interventions $[4,6,7]$. Among 563 ECMO patients who underwent 149 miscellaneous noncardiac surgical procedures, ECMO was associated with a $28 \%$ bleeding complication rate, without 
Table 2 Patient's preoperative and intraoperative characteristics

\begin{tabular}{|c|c|c|c|c|c|c|}
\hline \multirow[t]{2}{*}{ Characteristic } & \multicolumn{2}{|c|}{ Unmatched patients $(n=77)$} & \multirow[t]{2}{*}{$p$} & \multicolumn{2}{|c|}{ Propensity-matched patients $(n=42)$} & \multirow[t]{2}{*}{$p$} \\
\hline & ECMO $(n=35)$ & $\begin{array}{l}\text { non-ECMO } \\
(n=42)\end{array}$ & & ECMO $(n=21)$ & $\begin{array}{l}\text { non-ECMO } \\
(n=21)\end{array}$ & \\
\hline \multicolumn{7}{|l|}{ Preoperative } \\
\hline ICU admission to surgery interval (days) & $7(2-20)$ & $4(1-11)$ & 0.02 & $7(2-19)$ & $7(2-14)$ & 0.5 \\
\hline ECMO-to-surgery interval (days) & $7(2-15)$ & - & - & $7(2-18)$ & - & - \\
\hline Temperature $\left({ }^{\circ} \mathrm{C}\right)$ & $\begin{array}{l}37.1 \\
\quad(36.3-37.6)\end{array}$ & $36.8(36.5-37.7)$ & 0.79 & $\begin{array}{l}36.9 \\
\quad(36.4-37.7)\end{array}$ & $37.2(36.5-37.9)$ & 0.7 \\
\hline Renal replacement therapy & $24(69)$ & $22(52)$ & 0.17 & $12(57)$ & $11(52)$ & 1.0 \\
\hline Mechanical ventilation & $35(100)$ & $33(79)$ & 0.003 & $21(100)$ & $19(90)$ & 0.5 \\
\hline Inotrope score $(\mu \mathrm{g} / \mathrm{kg} / \mathrm{min})^{\mathrm{a}}$ & $32.5(6.4-68.3)$ & $21.5(0.0-85.1)$ & 0.50 & $26.7(5.5-59.8)$ & $22.2(1.2-119.0)$ & 0.9 \\
\hline Curative anticoagulation $^{\mathrm{b}}$ & $12(34)$ & $18(43)$ & 0.49 & $11(52)$ & $12(57)$ & 1.0 \\
\hline Bacteriemia & $16(46)$ & $18(43)$ & 0.82 & $12(57)$ & $7(33)$ & 0.2 \\
\hline Arterial $\mathrm{pH}$ & $\begin{array}{l}7.38 \\
\quad(7.35-7.43)\end{array}$ & $7.34(7.26-7.44)$ & 0.05 & $\begin{array}{l}7.40 \\
\quad(7.35-7.45)\end{array}$ & $7.36(7.28-7.43)$ & 0.3 \\
\hline Blood lactates $(\mathrm{mmol} / \mathrm{L})$ & $2.9(1.9-6.3)$ & $2.30(1.50-7.50)$ & 0.27 & $3.0(1.7-6.4)$ & $2.4(1.4-6.2)$ & 0.6 \\
\hline Bicarbonate (mmol/L) & $21(16-24)$ & $20(16-23)$ & 0.94 & $\begin{array}{l}20.4 \\
\quad(16.3-22.8)\end{array}$ & $20.5(16.3-22.6)$ & 0.9 \\
\hline Hemoglobin (g/dL) & $8.7(7.8-9.6)$ & $8.8(8.1-9.5)$ & 0.42 & $8.9(8.0-10.0)$ & $8.7(8.2-9.2)$ & 0.7 \\
\hline Platelet count (G/L) & $68(38-156)$ & $183(78-265)$ & $<0.001$ & $70(40-160)$ & $161(78-277)$ & 0.008 \\
\hline Prothrombin activity (\%) & $55(37-66)$ & $63(41-71)$ & 0.36 & $55(38-67)$ & $63(40-70)$ & 0.5 \\
\hline aPTT ratio & $3.8(2.4-6.6)$ & $1.3(1.1-1.9)$ & 0.13 & $1.3(1.2-1.9)$ & $1.3(1.1-1.9)$ & 0.5 \\
\hline Fibrinogen $(\mathrm{g} / \mathrm{L})$ & $3.8(2.40-6.6)$ & $5.1(4.2-7.3)$ & 0.04 & $3.3(2.2-6.4)$ & $5.1(4.2-7.3)$ & 0.1 \\
\hline \multicolumn{7}{|l|}{ Surgical approach } \\
\hline Laparotomy & $30(86)$ & $41(98)$ & 0.08 & $17(81)$ & $21(100)$ & 0.1 \\
\hline Other position & $5(14)$ & $1(2)$ & 0.09 & $4(19)$ & 0 & \\
\hline \multicolumn{7}{|l|}{ Type of procedure } \\
\hline Cholecystectomy & $8(23)$ & $11(26)$ & 0.80 & $5(24)$ & $6(29)$ & 1.0 \\
\hline Digestive ischemia & $15(43)$ & $17(40)$ & 1.00 & $8(38)$ & $10(48)$ & 0.8 \\
\hline Other & $12(34)$ & $14(33)$ & 1.00 & $8(38)$ & $5(24)$ & 0.3 \\
\hline \multicolumn{7}{|l|}{ Concomitant procedure } \\
\hline Stoma & $15(43)$ & $20(48)$ & 0.82 & $9(43)$ & $10(48)$ & 1.0 \\
\hline Digestive resection & $15(43)$ & $21(50)$ & 0.65 & $9(43)$ & $11(52)$ & 0.8 \\
\hline Vascular resection & $1(3)$ & 0 & 0.45 & 0 & 0 & 1.0 \\
\hline Patients transfused & $23(66)$ & $20(48)$ & 0.17 & $15(71)$ & $11(52)$ & 0.3 \\
\hline PRBCs & $2(0-4)$ & $0(0-2)$ & 0.008 & $2(0-3)$ & $0(0-2)$ & $\mathbf{0 . 0 3}$ \\
\hline FFP & $0(0-3)$ & $0(0-2)$ & 0.14 & $0(0-3)$ & $0(0-3)$ & 0.5 \\
\hline Platelets & $0(0-10)$ & $0(0-0)$ & $<0.001$ & $0(0-9)$ & $0(0-0)$ & 0.004 \\
\hline Bleeding & $10(29)$ & $4(10)$ & 0.04 & $6(29)$ & $1(5)$ & 0.09 \\
\hline
\end{tabular}

Results are expressed as number (\%) or median (first-third IQR)

aPTT activated partial thromboplastin time

Significant differences are in bold type

${ }^{a}$ Defined as dobutamine dose $+100 \times$ (epinephrine dose + norepinephrine dose), all in $\mu \mathrm{g} / \mathrm{kg} / \mathrm{min}$

${ }^{\mathrm{b}}$ Curative anticoagulation was defined as an unfractionated heparin dose $>12,000$ IU per day

bleeding episodes impacting in-ICU mortality [14]. A greater need for blood products was also demonstrated in two populations of patients receiving mechanical circulatory support, including 11 and 14 ECMO-treated patients, who had undergone miscellaneous noncardiac surgical interventions $[15,16]$. In another study, thoracotomy on ECMO led to unusual and uncontrollable massive hemorrhages [5]. In our cohort, 34\% of ECMO-treated patients 
Table 3 Patient's postoperative characteristics

\begin{tabular}{|c|c|c|c|c|c|c|}
\hline \multirow[t]{2}{*}{ Characteristic } & \multicolumn{2}{|c|}{ Unmatched patients $(n=77)$} & \multirow[t]{2}{*}{$p$} & \multicolumn{2}{|c|}{ Propensity-matched patients $(n=42)$} & \multirow[t]{2}{*}{$p$} \\
\hline & $\mathrm{ECMO}(n=35)$ & non-ECMO $(n=42)$ & & $\operatorname{ECMO}(n=21)$ & non-ECMO $(n=21)$ & \\
\hline \multicolumn{7}{|l|}{ Immediate postoperative period $(24 \mathrm{~h})$} \\
\hline Temperature $\left({ }^{\circ} \mathrm{C}\right)$ & $36.4(34-36.7)$ & $36.4(35.9-37.0)$ & 0.14 & $36.2(33.9-36.7)$ & $36.4(36.0-37.2)$ & 0.05 \\
\hline Renal replacement therapy & $26(74)$ & $23(55)$ & 0.09 & $14(67)$ & $11(52)$ & \\
\hline Inotrope score ( $\mathrm{pg} / \mathrm{kg} / \mathrm{min})$ & $42(14-87)$ & $25(10-45)$ & 0.12 & $42(10-97)$ & $25(7-74)$ & 0.6 \\
\hline Arterial $\mathrm{pH}$ & $7.34(7.28-7.44)$ & $7.32(7.25-7.40)$ & 0.31 & $7.31(7.26-7.43)$ & $7.30(7.24-7.39)$ & 0.6 \\
\hline Blood lactates (mmol/L) & $4(2-7)$ & $2.9(1.9-1.6)$ & 0.19 & $4.1(2.0-7.0)$ & $2.8(1.9-6.3)$ & 0.3 \\
\hline Bicarbonate $(\mathrm{mmol} / \mathrm{L})$ & $18(15-22)$ & $18(17-20)$ & 0.94 & $17(14-21)$ & $18(17-20)$ & 0.4 \\
\hline Hemoglobin $(\mathrm{g} / \mathrm{dL})$ & $9.5(7.8-10.1)$ & $10.2(9.2-11.4)$ & 0.002 & $9.5(8.7-10.3)$ & $9.9(9.2-10.9)$ & 0.4 \\
\hline Prothrombin activity $(\%)$ & $51(40-62)$ & $60(41-70)$ & 0.19 & $51(41-60)$ & $60(38-67)$ & 0.25 \\
\hline aPTT ratio & $1.5(1.2-1.7)$ & $1.3(1.0-1.8)$ & 0.32 & $1.4(1.2-1.6)$ & $1.3(1.0-2.0)$ & 0.5 \\
\hline Fibrinogen $(\mathrm{g} / \mathrm{L})$ & $2.9(1.9-5.1)$ & $4.8(3.6-6.6)$ & 0.002 & $2.7(2.0-3.6)$ & $4.8(3.5-6.7)$ & 0.006 \\
\hline Perioperative bleeding (72 h) & $26(74)$ & $14(33)$ & $<0.001$ & $15(71)$ & $8(38)$ & 0.04 \\
\hline \multicolumn{7}{|l|}{ Late postoperative period } \\
\hline Infection & $5(14)$ & $3(7)$ & 0.46 & $3(14)$ & $1(5)$ & 0.6 \\
\hline Bleeding & $12(34)$ & $1(2)$ & $<0.001$ & $6(29)$ & 0 & 0.02 \\
\hline Operative site & $4(33)$ & 0 & & $3(14)$ & 0 & \\
\hline Hemoperitoneum & $6(50)$ & 0 & & $2(10)$ & 0 & \\
\hline Stoma & $2(17)$ & $1(100)$ & & $1(5)$ & 0 & \\
\hline Reintervention & $10(29)$ & $4(10)$ & 0.04 & $5(24)$ & $1(5)$ & 0.2 \\
\hline Bleeding & $7(20)$ & $1(2)$ & 0.02 & $5(24)$ & 0 & 0.04 \\
\hline Infection & $3(9)$ & $3(7)$ & 1.00 & 0 & $1(5)$ & 1.0 \\
\hline Patients transfused & $27(77)$ & $16(38)$ & 0.001 & $20(95)$ & $17(81)$ & 0.3 \\
\hline Total PRBCs & $13(6-22)$ & $3(0-5)$ & $<0.001$ & $12(5-14)$ & $4(1-14)$ & 0.04 \\
\hline Total FFP & $9(3-17)$ & $0(0-4)$ & $<0.001$ & $9(3-16)$ & $0(0-10)$ & 0.005 \\
\hline Total platelets & $12(3-22)$ & $0(0-8)$ & $<0.001$ & $16(4-23)$ & $0(0-10)$ & 0.02 \\
\hline Mechanical ventilation duration (days) & $20(10-29)$ & $12(4-23)$ & 0.03 & $15(10-26)$ & $18(6-28)$ & 0.9 \\
\hline RRT duration (days) & $7(2-18)$ & $4(1-10)$ & 0.06 & $7(2-11)$ & $4(2-14)$ & 0.9 \\
\hline In-ICU death & $24(69)$ & $14(33)$ & 0.003 & $16(76)$ & $9(43)$ & 0.06 \\
\hline Perioperative mortality $(<72 \mathrm{~h})$ & $4(11)$ & $5(12)$ & 1.0 & 0 & $2(10)$ & 0.5 \\
\hline ICU stay (days) & $28(11-46)$ & $23(14-48)$ & 0.61 & $17(11-36)$ & $33(15-60)$ & 0.1 \\
\hline
\end{tabular}

Results are expressed as number (\%) or median (first-third IQR)

$a P T T$ activated partial thromboplastin time, $F F P$ fresh frozen plasma units, $P R B C s$ packed red blood cell units, $R R T$ renal replacement therapy Significant differences are in bold type

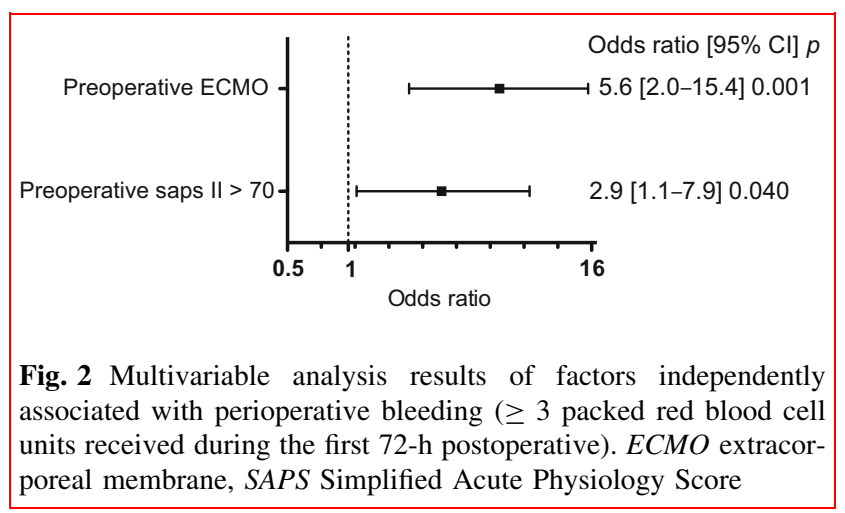

experienced postoperative bleeding, requiring reintervention in $20 \%$ of them and consumption of large amounts of blood products. However, perioperative mortality remained comparable to that of non-ECMO patients.

Patients on ECMO frequently develop complex coagulation disorders. Contact with the foreign circuit surfaces activates platelets and the coagulation cascade [12, 17-19], responsible for a hypercoagulation state. In turn, clots forming on the membrane trigger intense fibrinolysis, which, in addition to platelet consumption, is responsible for a secondary hypocoagulation state $[19,20]$. An acquired von Willebrand syndrome was also described 
under ECMO [21]. This pathophysiology opens an important area of research to limit the risk of bleeding during invasive procedures in this particular setting.

Intensivists need to evaluate more rigorous anticipation of those coagulation disorders by systematically replacing the ECMO circuit before surgery or providing greater quantities of blood products, particularly fibrinogen and platelets. Antifibrinolytic therapy with tranexamic acid achieved markedly lower bleeding rates in a large, multicenter, randomized trial during coronary artery surgery under cardiopulmonary bypass and could also be of interest during abdominal surgery [22]. Finally, better characterization of the patient's coagulation state using thromboelastometry and platelet aggregometry might also help optimize those patients' coagulation states in the future [23]. In our cohort, ECMO patients had lower presurgery platelet counts and fibrinogenemia than non-ECMO controls, consistent with more pronounced fibrinolysis. Whether or not a more aggressive protocol could manage coagulation disorders during invasive procedures might be a promising avenue of research.

For surgeons, coagulation disorders considerably modify the benefit/risk ratio of invasive surgery, with important potential implications. Bowel resection of a not viable segment might be prioritized over bowel preservation during ischemia, to avoid, as much as possible, the need for a second look. Systematic cholecystectomy should be reevaluated in this specific setting and should be avoided if it is not essential. Lastly, noninvasive approaches, like medical treatment of cholecystitis, should be preferred. Taken together, such complex interventions further emphasize the need to manage ECMO-treated patients in centers highly experienced in its medical and surgical aspects.

In our cohort, although ECMO patients' in-ICU mortality was very high, perioperative mortality and direct surgical complication-triggered mortality remained low compared to that of the non-ECMO group. It must be kept in mind that our patients predominantly had medical conditions with secondary abdominal complications. Although mortality-related reasons can be a matter of debate for this cohort, we think that the underlying disease and extreme severity of ECMO patients accounted, in large part, for the observed mortality. Notably, the cohort contained a high percentage of patients with refractory cardiogenic shock complicated by digestive ischemia, a pathology also associated with high mortality of non-ECMO patients.

Finally, our study has several limitations. Although it is the first homogeneous cohort of abdominal surgery procedures under ECMO, the sample size was small. Secondly, our cohort comprised selected medical ICU patients, limiting the external validity of our results. Lastly, our matching procedure used a propensity score to be ECMO- implanted to match ECMO and non-ECMO patients. Although this procedure yielded highly comparable groups, in terms of demographics and preoperative characteristics and the bleeding-event results for the matched cohort were consistent with multivariable analyses of the whole cohort, it cannot be excluded that factors associated with the patients' underlying conditions necessitating ECMO implantation may have accounted for the bleeding rate observed in matched ECMO patients.

We finally observed that the results of our study, undertaken to assess the outcomes of ECMO-treated patients who required emergency abdominal surgery, revealed that such invasive procedures were feasible, but associated with a markedly increased risk of hemorrhage. Further studies are needed to improve the management of such surgical interventions for patients on ECMO.

\section{Compliance with ethical standards}

Conflicts of interest The authors declare that they have no conflict of interest.

\section{References}

1. Combes A, Bréchot N, Luyt C-E et al (2012) What is the niche for extracorporeal membrane oxygenation in severe acute respiratory distress syndrome? Curr Opin Crit Care 18:527-532

2. Abrams D, Combes A, Brodie D (2014) Extracorporeal membrane oxygenation in cardiopulmonary disease in adults. J Am Coll Cardiol 63:2769-2778

3. Brodie D, Bacchetta M (2011) Extracorporeal membrane oxygenation for ARDS in adults. N Engl J Med 365:1905-1914

4. Zangrillo A, Biondi-Zoccai G, Landoni G et al (2013) Extracorporeal membrane oxygenation (ECMO) in patients with H1N1 influenza infection: a systematic review and meta-analysis including 8 studies and 266 patients receiving ECMO. Crit Care Lond Engl 17:R30

5. Marasco SF, Preovolos A, Lim K et al (2007) Thoracotomy in adults while on ECMO is associated with uncontrollable bleeding. Perfusion 22:23-26

6. Aubron C, Cheng AC, Pilcher D et al (2013) Factors associated with outcomes of patients on extracorporeal membrane oxygenation support: a 5-year cohort study. Crit Care Lond Engl 17:R73

7. Combes A, Leprince P, Luyt C-E et al (2008) Outcomes and long-term quality-of-life of patients supported by extracorporeal membrane oxygenation for refractory cardiogenic shock. Crit Care Med 36:1404-1411

8. Bréchot N, Luyt C-E, Schmidt M et al (2013) Venoarterial extracorporeal membrane oxygenation support for refractory cardiovascular dysfunction during severe bacterial septic shock. Crit Care Med 41:1616-1626

9. Extracorporeal Membrane Oxygenation for Severe Acute Respiratory Distress Syndrome (EOLIA trial). ClinicalTrials.gov NCT01470703

10. Aissaoui N, Luyt C-E, Leprince P et al (2011) Predictors of successful extracorporeal membrane oxygenation (ECMO) weaning after assistance for refractory cardiogenic shock. Intensive Care Med 37:1738-1745 
11. Lamarche Y, Chow B, Bédard A et al (2010) Thromboembolic events in patients on extracorporeal membrane oxygenation without anticoagulation. Innovations 5:424-429

12. Lubnow M, Philipp A, Dornia C et al (2014) D-dimers as an early marker for oxygenator exchange in extracorporeal membrane oxygenation. J Crit Care 29:473.e1-473.e5

13. D'Agostino RB (1998) Propensity score methods for bias reduction in the comparison of a treatment to a non-randomized control group. Stat Med 17:2265-2281

14. Taghavi S, Jayarajan SN, Mangi AA et al (2015) Examining noncardiac surgical procedures in patients on extracorporeal membrane oxygenation. ASAIO J Am Soc Artif Intern Organs $61: 520-525$

15. Taghavi S, Beyer C, Vora H et al (2014) Noncardiac surgery in patients on mechanical circulatory support. ASAIO J Am Soc Artif Intern Organs 60:670-674

16. Chestovich PJ, Kwon MH, Cryer HG et al (2011) Surgical procedures for patients receiving mechanical cardiac support. Am Surg 77:1314-1317

17. Cheung PY, Sawicki G, Salas E et al (2000) The mechanisms of platelet dysfunction during extracorporeal membrane oxygenation in critically ill neonates. Crit Care Med 28:2584-2590

18. Straub A, Wendel HP, Dietz K et al (2008) Selective inhibition of the platelet phosphoinositide 3-kinase p110beta as promising new strategy for platelet protection during extracorporeal circulation. Thromb Haemost 99:609-615
19. Malfertheiner MV, Philipp A, Lubnow M et al (2016) Hemostatic changes during extracorporeal membrane oxygenation: a prospective randomized clinical trial comparing three different extracorporeal membrane oxygenation systems. Crit Care Med 44:747-754

20. Dornia C, Philipp A, Bauer S et al (2015) D-dimers are a predictor of clot volume inside membrane oxygenators during extracorporeal membrane oxygenation. Artif Organs 39:782-787

21. Heilmann C, Geisen U, Beyersdorf F et al (2012) Acquired von Willebrand syndrome in patients with extracorporeal life support (ECLS). Intensive Care Med 38:62-68

22. Myles PS, Smith JA, Forbes A et al (2017) Tranexamic acid in patients undergoing coronary-artery surgery. $\mathrm{N}$ Engl J Med 376:136-148

23. Nair P, Hoechter DJ, Buscher $\mathrm{H}$ et al (2015) Prospective observational study of hemostatic alterations during adult extracorporeal membrane oxygenation (ECMO) using point-of-care thromboelastometry and platelet aggregometry. J Cardiothorac Vasc Anesth 29:288-296

Publisher's Note Springer Nature remains neutral with regard to jurisdictional claims in published maps and institutional affiliations. 